
United Nations Educational Scientific and Cultural Organization and International Atomic Energy Agency

THE ABDUS SALAM INTERNATIONAL CENTRE FOR THEORETICAL PHYSICS

\title{
RELATIVISTIC ION CYCLOTRON INSTABILITY DRIVEN BY MEV $\alpha$-PARTICLES IN NON-UNIFORM MAGNETIC FIELDS
}

\author{
J.Q. Dong 1 \\ Southwestern Institute of Physics, P.O. Box 432, Chengdu, 610041, People's Republic of China \\ and \\ The Abdus Salam International Centre for Theoretical Physics, Trieste, Italy, \\ and \\ K.R. Chen \\ National Cheng Kung University, Tainan 70101, China.
}

\begin{abstract}
The harmonic relativistic ion cyclotron instability driven by $\mathrm{Mev} \alpha$-particles in thermal deuterium plasmas confined by non-uniform magnetic fields is studied with gyrokinetic theory. An integral dispersion equation is derived and solved numerically. The results provide clear evidence for the existence of the instability of $\alpha$-particle cyclotron high harmonics in burning plasmas of one fast and one slow ion species. The characteristics of the modes are found to depend on magnetic non-uniformity parameter and $\alpha$-particle density rather strongly.
\end{abstract}

MIRAMARE - TRIESTE

July 2004

\footnotetext{
${ }^{1}$ Regular Associate of the Abdus Salam ICTP.
} 


\section{INTRODUCTION}

The relativistic electron cyclotron instability in magnetized plasmas has been an active research area for the last four decades. The mechanism is significantly appreciated and widely applied in fusion research, space plasma, as well as in microwave generation technology. On the other hand, the concept of relativistic ion cyclotron instability was suggested by Chen a few years ago $^{1-3}$ and studied more in detail recently. ${ }^{4}$ It was shown with particle in cell simulation and with local gyrokinetic theory that relativistic ion cyclotron instability may occur in a variety of plasma systems due to the difference of mass deficits between fast and slow ions. While the studies on this subject provide an opportunity to explore novel mechanism for coherent wave emission, the importance of the studies is to explore means for energy extraction from fusion produced Mev $\alpha$-particles through plasma wave excitation or amplification at the expense of energetic $\alpha$-particles. The latter is one of the major concerns in burning fusion plasmas and, therefore, contracts considerable research attentions. ${ }^{5-7}$ Nevertheless, most of the past studies were performed for plasmas in uniform magnetic field configurations. Therefore, the effect of magnetic field non-uniformity is one of the major issues raised when the theory is applied to realistic laboratory and space plasma systems where magnetic fields are truly non-uniform.

The harmonic relativistic ion cyclotron instability is studied with gyrokinetic theory in nonuniform magnetic field configurations in this work. An integral dispersion equation is derived and a computer code for solving the equation is developed. Preliminary numerical results are obtained and provide the evidence for the existence of the instability in plasmas of one fast and one slow ion species when magnetic fields are non-uniform. In Sec. 2, we introduce the physical model employed and the basic eigenmode equation obtained in a non-uniform magnetic

geometry. Numerical results are presented in Sec. 3. Finally, conclusions of the present study and discussion are given in Sec. 4.

\section{PHYSICAL MODEL AND EIGENMODE EQUATION}

In a non-uniform magnetic geometry, the equilibrium magnetic field is written as

$$
\mathbf{B}=B_{0}\left[1+b_{1}(x)\right] \mathbf{z},
$$

with

$$
L_{B}=\left[\frac{d b_{1}(x)}{d x}\right]^{-1} \gg \frac{v_{\perp}}{\Omega}, \lambda,
$$

where $L_{B}$ is the measure of the magnetic non-uniformity, $v_{\perp}$ and $\Omega$ are the perpendicular velocity component and the gyro-frequency for charged particles, respectively.

The particle orbit to the lowest order in $L_{B}^{-1}$ may be written as

$$
x^{\prime}-x=-\frac{v_{\perp}}{\omega_{c}}\left[\sin \left(\theta-\omega_{c} \tau\right)-\sin \theta\right]
$$




$$
y^{\prime}-y=\frac{v_{\perp}}{\omega_{c}}\left[\cos \left(\theta-\omega_{c} \tau\right)-\cos \theta\right]+u_{d} \tau
$$

and

$$
z^{\prime}-z=v_{\|} \tau
$$

where

$$
\begin{gathered}
\omega_{c}=\omega_{0}\left[1+b_{1}\left(x_{g}\right)\right], \quad \omega_{0}=\frac{q B_{0}}{m c \gamma}, \\
x_{g}=x+\frac{v_{\perp}}{\omega_{0}} \sin \theta, \quad u_{d}=-\frac{\gamma v_{\perp}^{2}}{2 \Omega L_{B}}, \\
\gamma=\frac{1}{\sqrt{1-\frac{v^{2}}{c^{2}}}}=\sqrt{1+\frac{p^{2}}{m^{2} c^{2}}}, \quad \Omega=\frac{q B}{m c} .
\end{gathered}
$$

All the symbols have their usual meaning such as $m$ is the rest mass, $q$ is the charge, $c$ is the speed of light in vacuum, $p$ is momentum. $x_{g}$ is the position of the particle guiding center, that is a constant of motion. $u_{d}$ is the magnetic gradient drift velocity with the relativistic effect included.

Electrostatic perturbations are considered only with,

$$
\mathbf{E}=-\nabla \tilde{\phi}
$$

and

$$
\tilde{\phi}(\mathbf{r}, t)=\tilde{\phi}(x) e^{-i\left(\omega t-k_{y}-k_{\|} z\right)}
$$

From the linearized Vlasov's Equation, it is easy to get the perturbation of distribution function as

$$
\tilde{f}_{1}(\mathbf{r}, \mathbf{p}, t)=q \int_{-\infty}^{t} \nabla^{\prime} \tilde{\phi}\left(\mathbf{r}^{\prime}, t^{\prime}\right) \cdot \nabla p f_{0}(\mathbf{p}) d t^{\prime}
$$

where $f_{0}$ is the equilibrium distribution function, $\mathbf{r}$ is the position in real space. Introducing

$$
\tilde{f}_{1}(\mathbf{r}, \mathbf{p}, t)=\tilde{f}_{1}(x, \mathbf{p}) e^{-i \omega t+i k_{y} y+i k_{\|} z} .
$$

then

$$
\tilde{f}_{1}(x, \mathbf{p})=q \int_{-\infty}^{t}\left[\left(\mathbf{e}_{x} \frac{d}{d x^{\prime}}+i k_{y} \mathbf{e}_{y}\right) \tilde{\phi}\left(x^{\prime}\right) \cdot \nabla_{p} f_{0}\right] e^{-i \omega\left(t^{\prime}-t\right)+i k_{y}\left(y^{\prime}-y\right)+i k_{\|}\left(z^{\prime}-z\right)} d t^{\prime}
$$

The perturbation of density in real space as a function of $x$ is

$$
\tilde{n}_{1}(x)=\int \tilde{f}_{1}(x, \mathbf{p}) d \mathbf{p}
$$

Performing Fourier transform for both $\tilde{\phi}(x)$ and $\tilde{n}_{1}(x)$,

$$
\tilde{g}(x)=\frac{1}{\sqrt{2 \pi}} \int_{-\infty}^{\infty} \widehat{g}(k) e^{i k x} d k,
$$


where $g$ represents $\phi$ or $n_{1}$, then the density perturbation in k-space is obtained as

$$
\begin{gathered}
\widehat{n}_{1}(k)=\frac{2 \pi q}{m} \sum_{l} \frac{1}{\sqrt{2 \pi}} \int_{-\infty}^{+\infty} d k^{\prime} \frac{1}{\sqrt{2 \pi}} \int_{-\infty}^{+\infty} d x e^{i\left(k^{\prime}-k\right) x} \widehat{\phi}\left(k^{\prime}\right) \times \\
\int p_{\perp} d p_{\perp} d p_{\|} f_{0}\left\{\frac{k_{\perp} k_{\perp}^{\prime}}{2 \Omega \omega_{r e}}\left[J_{l-1}(\alpha) J_{l-1}\left(\alpha^{\prime}\right)-J_{l+1}(\alpha) J_{l+1}\left(\alpha^{\prime}\right)\right]+\right. \\
\left.\frac{1}{\left(\gamma \omega_{r e}\right)^{2}} J_{l}(\alpha) J_{l}\left(\alpha^{\prime}\right)\left[\gamma k_{\|}^{2}-\frac{\gamma l k_{y}}{L_{B}}-\frac{1}{\gamma c^{2}}\left(\frac{k_{\|} p_{\|}}{m}+l \Omega\right)^{2}-\frac{k_{y} u_{d}}{c^{2}}\left(\frac{k_{\|} p_{\|}}{m}+l \Omega\right)\right]\right\} e^{i l\left(\psi^{\prime}-\psi\right)},
\end{gathered}
$$

where

$$
\begin{aligned}
\alpha & =\frac{p_{\perp} k_{\perp}}{m \Omega}, \quad k_{\perp}^{2}=k^{2}+k_{y}^{2}, \\
\alpha^{\prime} & =\frac{p_{\perp} k_{\perp}^{\prime}}{m \Omega}, \quad k_{\perp}^{\prime 2}=k^{2}+k_{y}^{2}, \\
\omega_{r e} & =\omega-\frac{k_{\|} p_{\|}}{\gamma m}-\frac{l \Omega}{\gamma}-k_{y} u_{d}, \\
\sin \psi & =\frac{k_{y}}{k}, \quad \sin \psi^{\prime}=\frac{k_{y}}{k^{\prime}},
\end{aligned}
$$

$J_{n}$ is Bessel function of order $n$ with $n=l-1, l, l+1$. The terms with the factor $\left(k_{\|} p_{\|} / m+l \Omega\right)$ represent the contributions from relativistic effects.

Poisson's Equation

$$
\nabla^{2} \phi(\mathbf{r})=-4 \pi \sum_{\beta} q_{\beta} \tilde{n}_{\beta}(\mathbf{r})
$$

is converted into the $\mathrm{k}$-space as

$$
\left(k_{y}^{2}+k^{2}+k_{\|}^{2}\right) \widehat{\phi}(k)=4 \pi \sum_{\beta} q_{\beta} \widehat{n}_{\beta}(k) .
$$

Substituting Eq. (13) into Eq. (15), we get the integral dispersion equation

$$
\left(k_{y}^{2}+k^{2}+k_{\|}^{2}\right) \widehat{\phi}(k)=\int_{-\infty}^{\infty} \frac{d k^{\prime}}{\sqrt{2 \pi}} K\left(k, k^{\prime}\right) \widehat{\phi}\left(k^{\prime}\right)
$$

where

$$
\begin{gathered}
K\left(k, k^{\prime}\right)=2 \pi \sum_{\beta} \frac{\omega_{p \beta}^{2}}{\sqrt{2 \pi}} \int_{-\infty}^{+\infty} d x e^{i\left(k^{\prime}-k\right) x} \times \\
\sum_{l} e^{i l\left(\psi-\psi^{\prime}\right)} \int p_{\perp} d p_{\perp} d p_{\|} f_{0}\left\{\frac{k_{\perp} k_{\perp}^{\prime}}{2 \Omega \omega_{r e}}\left[J_{l-1}(\alpha) J_{l-1}\left(\alpha^{\prime}\right)-J_{l+1}(\alpha) J_{l+1}\left(\alpha^{\prime}\right)\right]+\right. \\
\left.\frac{1}{\left(\gamma \omega_{r e}\right)^{2}} J_{l}(\alpha) J_{l}\left(\alpha^{\prime}\right)\left[\gamma k_{\|}^{2}-\frac{\gamma l k_{y}}{L_{B}}-\frac{1}{\gamma c^{2}}\left(\frac{k_{\|} p_{\|}}{m}+l \Omega\right)^{2}-\frac{k_{y} u_{d}}{c^{2}}\left(\frac{k_{\|} p_{\|}}{m}+l \Omega\right)\right]\right\}
\end{gathered}
$$

$\omega_{p \beta}$ is the plasma frequency of species $\beta$.

The dispersion equation, Eq. (16) may be simplified with a few limits. In the limit of $k_{y}=0$, we have $\psi=\psi^{\prime}=0, k_{\perp}^{2}=k^{2}$ and $k_{\perp}^{\prime 2}=k^{\prime 2}$. In addition, the magnetic gradient drift effects 
disappear. The equation may be further simplified with the assumption of $k_{\|}=0$, and therefore $\omega_{r e}=\omega-l \Omega / \gamma$. In this case, the integral equation becomes

$$
k^{2} \widehat{\phi}(k)=\int_{-\infty}^{\infty} \frac{d k^{\prime}}{\sqrt{2 \pi}} K\left(k, k^{\prime}\right) \widehat{\phi}\left(k^{\prime}\right)
$$

where

$$
\begin{gathered}
K\left(k, k^{\prime}\right)=\sum_{\beta} K_{\beta}\left(k, k^{\prime}\right)=2 \pi \sum_{\beta} \omega_{p \beta}^{2} \frac{1}{\sqrt{2 \pi}} \int_{-\infty}^{+\infty} d x e^{i\left(k^{\prime}-k\right) x} \sum_{l} \int p_{\perp} d p_{\perp} d p_{\|} f_{0} \times \\
\left\{\frac{k_{\perp} k_{\perp}^{\prime}}{2 \Omega \omega_{r e}}\left[J_{l-1}(\alpha) J_{l-1}\left(\alpha^{\prime}\right)-J_{l+1}(\alpha) J_{l+1}\left(\alpha^{\prime}\right)\right]-\frac{(l \Omega)^{2}}{\gamma^{3}\left(\omega_{r e} c\right)^{2}} J_{l}(\alpha) J_{l}\left(\alpha^{\prime}\right)\right\} .
\end{gathered}
$$

The species subscript $\beta$ is omitted in part of the above equations.

Now, we calculate the contributions from each species.

For electrons, $\alpha=\alpha^{\prime} \simeq 0$ and

$$
J_{l}(0)= \begin{cases}1 & \text { if } l=0 \\ 0 & \text { otherwise }\end{cases}
$$

The integration over velocity space gives $1 / 2 \pi$, then, the terms left are

$$
\frac{k k^{\prime}}{2 \Omega_{e}}\left[\frac{1}{\omega-\Omega_{e}}-\frac{1}{\omega+\Omega_{e}}\right]=\frac{k k^{\prime}}{\omega^{2}-\Omega_{e}^{2}} \simeq-\frac{k k^{\prime}}{\Omega_{e}^{2}},
$$

since $\Omega_{e} \gg \omega$.

The contribution from the electrons to the kernel $K\left(k, k^{\prime}\right)$ is

$$
K_{e}\left(k, k^{\prime}\right)=-k \frac{\omega_{p e}^{2}}{\Omega_{e 0}^{2}} \frac{k^{\prime}}{\sqrt{2 \pi}} \int_{-\infty}^{+\infty} d x e^{i\left(k^{\prime}-k\right) x} \frac{1}{\left[1+b_{1}(x)\right]^{2}} .
$$

For the slow ions with Maxwellian velocity distribution, we have

$$
\gamma_{s} \simeq 1, \quad \omega_{r e s} \simeq \omega-l_{s} \Omega_{s}
$$

and

$$
\int_{0}^{\infty} d x e^{-\lambda x} J_{l}(2 \alpha \sqrt{x}) J_{l}\left(2 \alpha^{\prime} \sqrt{x}\right)=\frac{1}{\lambda} I_{l}\left(\frac{2 \alpha \alpha^{\prime}}{\lambda}\right) \exp \left(-\frac{\alpha^{2}+\alpha^{\prime 2}}{\lambda}\right),
$$

where $I_{l}$ is the modified Bessel function of order $l$. Therefore, the contribution from the slow ions is

$$
\begin{aligned}
K_{s}\left(k, k^{\prime}\right) & =\omega_{p s}^{2} \int_{-\infty}^{+\infty} \frac{d x}{\sqrt{2 \pi}} e^{i\left(k^{\prime}-k\right) x} \frac{2 l_{s} \Omega_{s}}{\omega_{r e s} v_{t s}^{2}}\left(1-\frac{l_{s} \Omega_{s}}{2 \omega_{r e s}} \frac{v_{t s}^{2}}{c^{2}}\right) I_{l_{s}}\left(\frac{k k^{\prime} v_{t s}^{2}}{2 \Omega_{s}^{2}}\right) \exp \left(-\frac{v_{t s}^{2}\left(k^{2}+k^{\prime 2}\right)}{4 \Omega_{s}^{2}}\right) \\
& +\sqrt{2 \pi} \delta\left(k-k^{\prime}\right) \frac{\omega_{p s}^{2}}{v_{t s}^{2}} \frac{4}{l_{f}^{2}\left(Z_{f} m_{s} / m_{f} Z_{s}\right)^{2}-1} I_{1}\left(\frac{k k^{\prime} v_{t s}^{2}}{2 \Omega_{s}^{2}}\right) \exp \left(-\frac{\left(k^{2}+k^{\prime 2}\right) v_{t s}^{2}}{4 \Omega_{s}^{2}}\right) .
\end{aligned}
$$


The second part of Eq. (23) is the contribution from the first harmonic after neglecting the second term in Eq. (18).

The integration over velocity space for the fast ions with isotropic velocity distribution function $f_{0}=\delta\left(p-p_{0}\right) / 4 \pi p_{0}^{2}$ is performed analytically and results in,

$$
\begin{gathered}
2 \pi \int p_{\perp} d p_{\perp} d p_{\|} f_{0}\left\{\frac{k_{\perp} k_{\perp}^{\prime}}{2 \Omega_{f} \omega_{r e}}\left[J_{l_{f}-1}(\alpha) J_{l_{f}-1}\left(\alpha^{\prime}\right)-J_{l_{f}+1}(\alpha) J_{l_{f}+1}\left(\alpha^{\prime}\right)\right]\right. \\
\left.-\frac{\left(l_{f} \Omega_{f}\right)^{2}}{\left(\gamma_{f} \omega_{r e}\right)^{2} \gamma_{f} c^{2}} J_{l_{f}}(\alpha) J_{l_{f}}\left(\alpha^{\prime}\right)\right\}=\frac{1}{2} \int_{0}^{\pi}\left\{\frac { k k ^ { \prime } } { 2 \Omega _ { f } \omega _ { r e f } } \left[J_{l_{f}-1}\left(\alpha_{0}\right) J_{l_{f}-1}\left(\alpha_{0}^{\prime}\right)-\right.\right. \\
\left.\left.J_{l_{f}+1}\left(\alpha_{0}\right) J_{l_{f}+1}\left(\alpha_{0}^{\prime}\right)\right]-\frac{\left(l_{f} \Omega_{f}\right)^{2}}{\gamma_{f}^{3}\left(\omega_{r e f} c\right)^{2}} J_{l_{f}}\left(\alpha_{0}\right) J_{l_{f}}\left(\alpha_{0}^{\prime}\right)\right\} \sin \theta d \theta
\end{gathered}
$$

where

$$
\alpha_{0}=\frac{p_{0} k}{m_{f} \Omega_{f}} \sin \theta, \quad \alpha_{0}^{\prime}=\frac{p_{0} k^{\prime}}{m_{f} \Omega_{f}} \sin \theta
$$

are taken the values at $x=0$ surface as an approximation.

Consequently, the contribution from the fast ions to the kernel is

$$
\begin{gathered}
K_{f}\left(k, k^{\prime}\right)=\frac{\omega_{p f}^{2}}{2 \sqrt{2 \pi}} \int_{-\infty}^{+\infty} d x e^{i\left(k^{\prime}-k\right) x} \sum_{l_{f}} \int_{0}^{\pi}\left\{\frac { k k ^ { \prime } } { 2 \Omega _ { f } \omega _ { r e f } } \left[J_{l_{f}-1}\left(\alpha_{0}\right) J_{l_{f}-1}\left(\alpha_{0}^{\prime}\right)-\right.\right. \\
\left.\left.J_{l_{f}+1}\left(\alpha_{0}\right) J_{l_{f}+1}\left(\alpha_{0}^{\prime}\right)\right]-\frac{\left(l_{f} \Omega_{f}\right)^{2}}{\gamma_{f}^{3}\left(\omega_{r e f} c\right)^{2}} J_{l_{f}}\left(\alpha_{0}\right) J_{l_{f}}\left(\alpha_{0}^{\prime}\right)\right\} \sin \theta d \theta,
\end{gathered}
$$

where $\omega_{r e f}=\omega-l_{f} \Omega_{f} / \gamma_{f}$.

\section{NUMERICAL RESULTS}

The integral dispersion equation, Eq. (18), has to be solved numerically. The schemes developed in the studies of drift instabilities are employed and the computer code HD7 is modified for the

purpose. ${ }^{8}$ The parameters used are the same as that in Ref. 3. The energetic $\alpha$-particles have energy of $3.5 \mathrm{Mev}$ without a preferential direction in momentum space. The rest mass of an $\alpha$-particle is $7294.2 m_{e}$. The slow deuterium ions have temperature of $5 \mathrm{kev}$ and rest mass of $3670.2 m_{e}$. The background magnetic field $B_{0}=5 \mathrm{~T}$. The deuteron density is $n_{D}=10^{13} \mathrm{~cm}^{-3}$. The high harmonic $l_{f}=13$ quadratic instability is considered only.

The magnetic field is assumed to be parabolic for simplicity,

$$
B(x)=B_{0}\left(1+\varepsilon_{b} x^{2}\right),
$$

where the parameter $\varepsilon_{b}$ is the measure of magnetic non-uniformity.

The typical eigenfunctions $\hat{\phi}(k)(a)$ and $\phi(x)(b)$ in $k$-and $x$-spaces are shown in Fig. 1. The parameters for the magnetic gradient and $\alpha$-particle density are $\varepsilon_{b}=0.132 \times 10^{-2}$ and 
$n_{f} / n_{e}=0.4485 \times 10^{-2}$, respectively. The heavy and light lines are the real and imaginary parts, respectively. It is clearly shown that the modes are confined in limited regions in both the wave vector and coordinate spaces. The maximum in $\mathrm{k}$-space around $k \simeq 13$ is close to the local results while the stable gaps there disappear here. ${ }^{1,2}$

The normalized growth rate $(a)$ and real frequency $(b)$ as function of the magnetic nonuniformity parameter $\varepsilon_{b}$ are given in Fig. 2 for $n_{f} / n_{e}=0.998 \times 10^{-3}$ (the light lines) and $0.4485 \times 10^{-2}$ (the heavy lines), respectively. Here, $\Delta \hat{\omega}_{r}=\omega_{r} / \Omega_{f 0}-l_{f}$ is shift of the normalized frequency from the harmonic number. The numbers here are of the same order of magnitude as what were obtained from local analysis. ${ }^{1,2}$ However, the differences between the local and non-local analyses are clear. There are no eigenmode structures and, therefore, no unstable modes in the non-local analysis when the magnetic non-uniformity goes to zero while the results obtained with the local theory clearly show the existence of the instability in uniform magnetic fields. This is understandable since the eigenmode equation can only govern localized modes while the local treatment is fairly suitable for uniform cases. On the other hand, The eigenmode structures disappear and the modes become stable when the magnetic non-uniformity goes too high due to the resonance characteristics of the cyclotron instability. It is also shown that the mode growth rate increases when the fast ion density decreases for low and intermediate magnetic non-uniformity $\left(\varepsilon_{b} \leq 0.0016\right)$ while the dependence seems rather weak for high nonuniformity. In addition, it seems that there is an optimum non-uniformity parameter for each value of density ratio $n_{f} / n_{e}$, at which the growth rate reaches a maximum. Here, the optimum $\varepsilon_{b}$ 's are 0.001 and 0.00176 for $n_{f} / n_{e}=0.998 \times 10^{-3}$ and $0.4485 \times 10^{-2}$, respectively.

The normalized growth rate $(a)$ and real frequency $(b)$ as function of the $\alpha$-particle density are given in Fig. 3 for $\varepsilon_{b}=1.075 \times 10^{-03}$ (the light lines) and $1.32 \times 10^{-03}$ (the heavy lines), respectively. It is reported from the local theory that, for the sixteenth $\alpha$-particle harmonic, the mode maximum growth rate (with respect to $k_{x}$ ) is proportional to the square root of $\alpha$ particle density while its corresponding wave number is not sensitive. ${ }^{3}$ Here, the mode growth rate increases with $\alpha$-particle density only in the parameter regime $n_{f} / n_{e} \leq 0.002$. It saturates and starts to decrease for further increasing of $\alpha$-particle density. The maximum growth rates occur at $n_{f} / n_{e} \simeq 0.2198 \times 10^{-2}$ and $0.2433 \times 10^{-2}$ for $\varepsilon_{b}=1.075 \times 10^{-2}$ and $1.32 \times 10^{-2}$ ,respectively. The decreasing of the growth rate for higher fast ion density is in agreement with the local analysis which indicates that the cyclotron waves at certain wavelengths become stable as the fast ion density goes higher. ${ }^{3}$ It is also shown that the growth rate for $\varepsilon_{b}=1.075 \times 10^{-2}$ is higher than that for $\varepsilon_{b}=1.32 \times 10^{-2}$ when $n_{f} / n_{e} \leq 0.15 \times 10^{-2}$ and vice versa although the difference is not significant. 


\section{Conclusions and discussion}

The relativistic ion cyclotron instabilities at high harmonics driven by Mev $\alpha$-particles are studied with gyrokinetic theory in non-uniform parabolic magnetic fields of a slab geometry. The existence of the modes in such configurations is demonstrated. It is clearly shown that the modes with limited wave vector spectrums are confined in limited regions of the real space. In addition, there seems to be an optimum non-uniformity parameter for each value of density ratio $n_{f} / n_{e}$, at which the growth rate reaches a maximum. The growth rate decays rather rapidly away from the optimum non-uniformity.

For fixed magnetic non-uniformity, the mode growth rate increases with $\alpha$-particle density in the parameter regime $n_{f} / n_{e} \leq 0.002$. It saturates and starts to decrease for further increasing of $\alpha$-particle density. The maximum growth rates occur at $n_{f} / n_{e} \simeq 0.2198 \times 10^{-2}$ and $0.2433 \times 10^{-2}$ for $\varepsilon_{b}=1.075 \times 10^{-2}$ and $1.32 \times 10^{-2}$, respectively.

Eigenfunction structures have not been found for negative $\varepsilon_{b}$ and linear $\left(B=B_{0}\left(1+\varepsilon_{b} x\right)\right.$ configurations although attempt has been made. One possibility for such failure is that eigenmodes do not exist at all in such configurations due to physical reasons. The other is numerical issues including appropriate guess eigenvales provided for the code. The linear configurations are more relevant to tokamak devices and are worthwhile for further investigation. Such works are in progress and results will be presented in the near future.

Acknowledgments. One of the authors (JQD) is a Regular Associate of the Abdus Salam International Centre for Theoretical Physics, trieste, Italy. He appreciates the hospitality of the Centre, extended to him during his visit. He also thanks Academician Li, Zhengwu for continual encouragements. The work is supported, in part, by the National Natural Science Foundation of China under Grant No. 10135020. 


\section{References}

1. K.R. Chen, Phys. Rev. Lett. 73, 3534 (1994).

2. K.R. Chen, W. Horton, and J.W. Van Dam, Phys. Plasmas 1, 1195(1994).

3. K.R. Chen, Phys. Plasmas 7, 844 (2000); 7857 (2000).

4. K.R. Chen, Phys. Plasmas 10, 1315 (2003).

5. A. Fruchtman, N.J. Fisch and E.J. Valeo, Phys. Plasmas 4, 138 (1997).

6. N.J. Fisch and Jean-Marcel Rax, Phys. Rev. Lett. 69, 612 (1992).

7. E.J. Valeo and N.J. Fisch, Phys. Rev. Lett. 73, 3536 (1994).

8. J.Q. Dong, W. Horton, Phys. Fluids B5, 1581 (1993). 

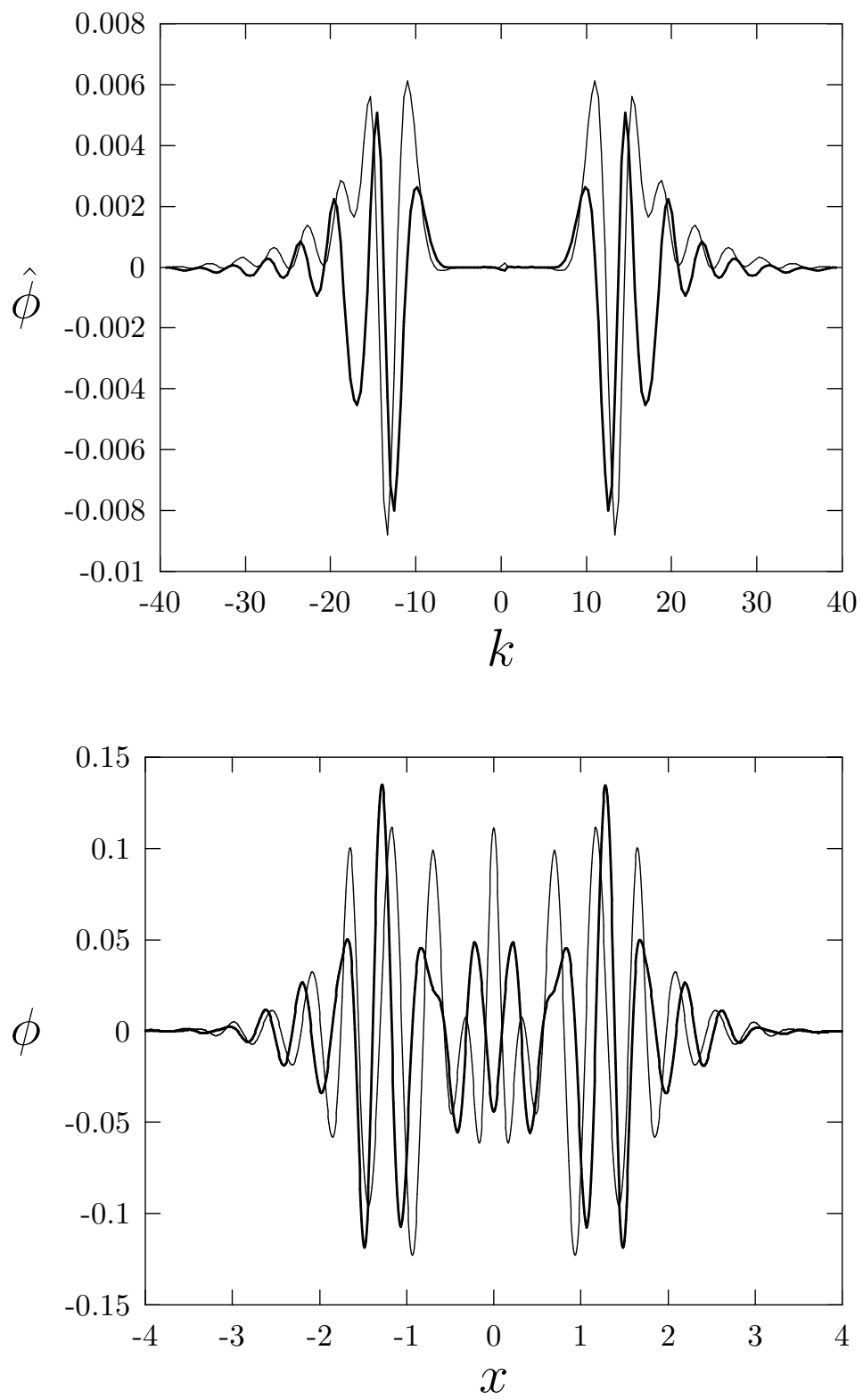

Fig.1. Eigenfunctions in wavevector $(a)$ and real $(b)$ spaces. The parameters are $\varepsilon_{b}=0.132 \times 10^{-2}$ and $n_{f} / n_{e}=$ $0.4485 \times 10^{-2}$ besides the parameter values mentioned in the text. The heavy and the light lines are the real imaginary parts, respectively. 

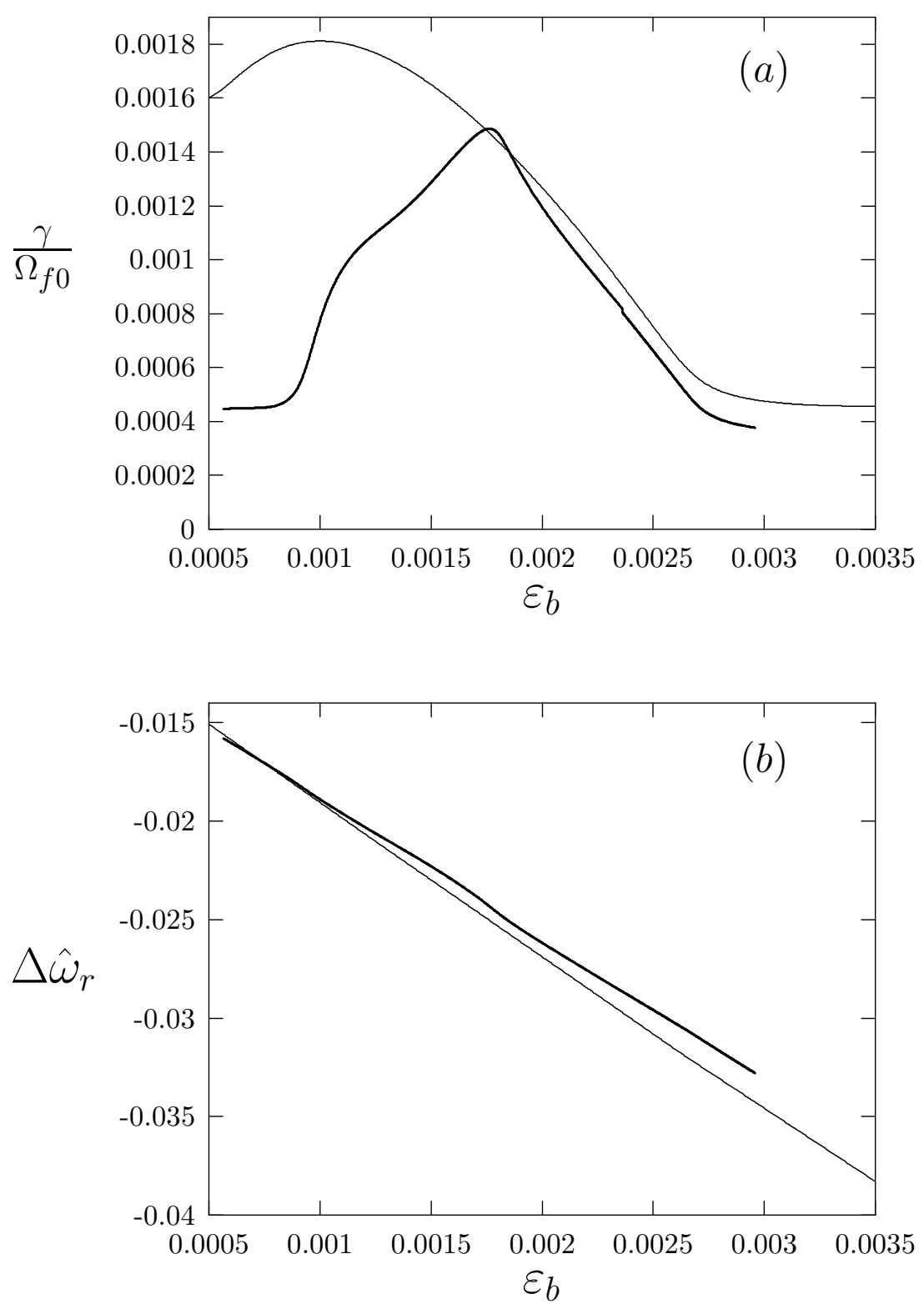

Fig. 2. The growth rate $(a)$ and the shift of the normalized real frequency from the harmonic number $(b)$ as functions of magnetic non-uniformity parameter $\varepsilon_{b}$ for $n_{f} / n_{e}=0.998 \times 10^{-3}$ (the light lines) and $0.445 \times 10^{-2}$ (the heavy lines), respectively. 

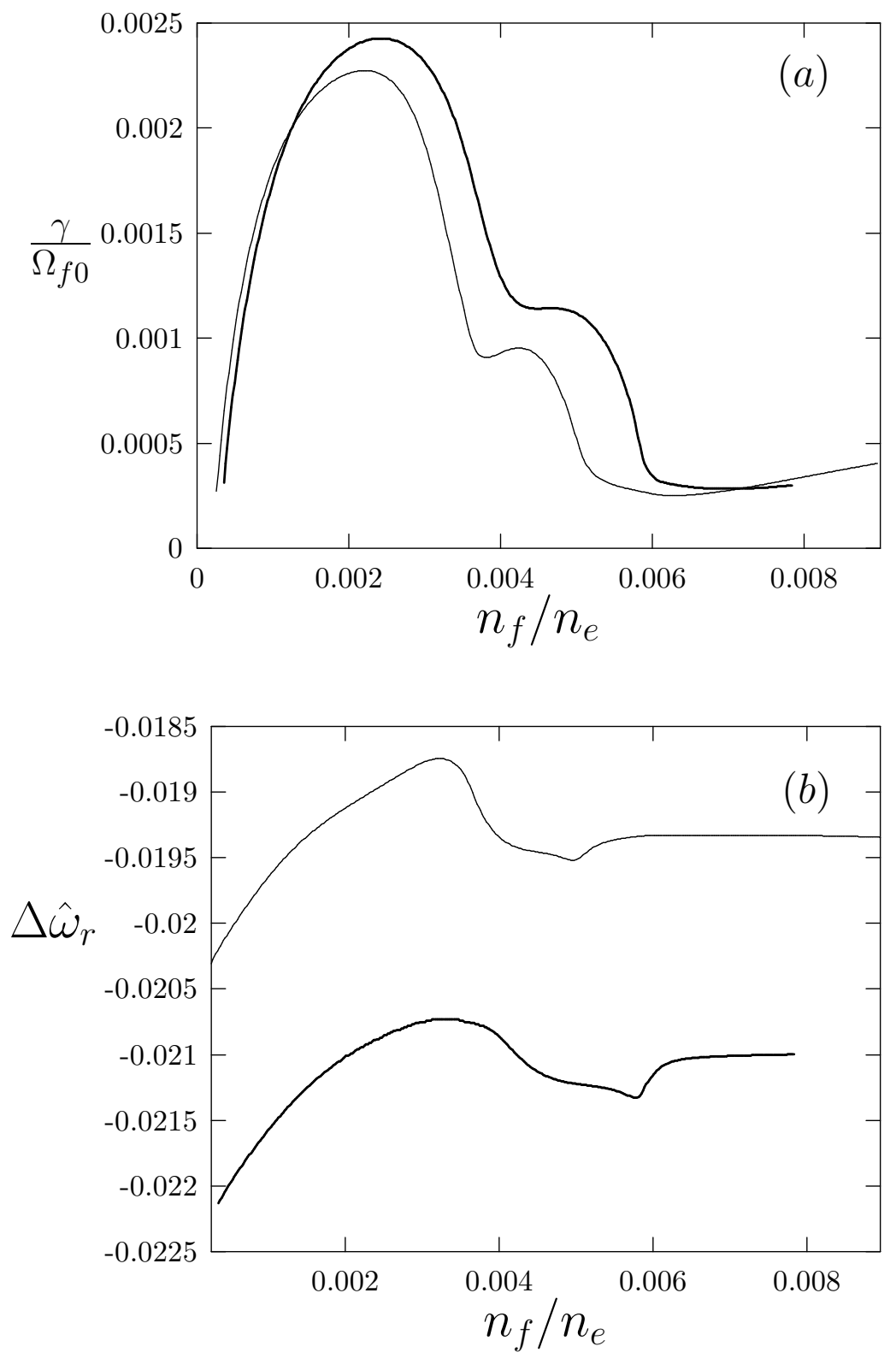

Fig.3. The growth rate $(a)$ and the shift of the normalized real frequency from the harmonic number $(b)$ as functions of fast ion density for magnetic non-uniformity parameter $\varepsilon_{b}=1.32 \times 10^{-2}$ (the heavy lines) and $1.075 \times 10^{-2}$ (the light lines), respectively. 